\title{
Association of metabolic syndrome in polycystic ovarian syndrome
}

\author{
Pooja Jeengar, Madhubala Chauhan
}

Correspondence: Dr Pooja Jeenger, PG Student in Department of Obstetrics \& Gynaecology, RNT Medical College, Udaipur, Rajasthan; Emailpoojajeengar@rediffmail.com

Distributed under Creative Commons Attribution-Share Alike 4.0 International.

\begin{abstract}
Objective: The aim of the study is to observe the association of metabolic syndrome in Polycystic Ovarian Syndrome (PCOS). Materials and Methods: A group of 50 women having documented features of PCOS (according to Rotterdam criteria 2003) underwent detailed evaluation for presence of metabolic syndrome (according to NCEP criteria) and compared with 50 women having normal pelvic study. Result: Twenty nine $(58 \%)$ out of 50 PCOS women met criteria of metabolic syndrome as compared to $36 \%$ patients of control group. Waist circumference, systolic and diastolic blood pressures, fasting glucose had a significantly higher value among those with PCOS in comparison to those without the PCOS. Conclusion: The metabolic syndrome and its individual components are common in PCOS, particularly among obese women.
\end{abstract}

Keywords: Polycystic ovarian syndrome, metabolic syndrome.

Polycystic ovarian syndrome (PCOS) is the most common form of anovulatory infertility. It is also one of most common endocrine disorder in women of reproductive age, affecting $5 \%$ to $10 \%$ of women worldwide. It is characterized by combination of hyperandrogenism (either clinical or bio - chemical), chronic anovulation and polycystic ovaries [1]. PCOS is frequently associated with insulin resistance and obesity [2]. Affected women seem to have a form of the metabolic syndrome that manifest itself in early adult life with gynecological symptoms. The etiology of PCOS is uncertain. The most common cause of insulin resistance and compensatory hyperinsulinemia is obesity [3]. Despite insulin resistance in adipose and skeletal muscle, the ovary remains relatively sensitive to insulin. Potentially a gene or series of genes renders the ovaries susceptible to insulin [4-8]. Both insulin and insulin like growth factor 1 along with increased $\mathrm{LH}$ have stimulatory effect on thecal cells leading to thecal hyperplasia, increased androgen secretion, arrest of follicular development and therefore, anovulation along with menstrual disturbances. Insulin also acts on liver to inhibit production of sex hormone binding globulin (SHBG) and insulin like growth factor-1 (IGF-1). This leads to increase in biologically available free testosterone. Thus insulin resistance not only increases

Received: $25^{\text {th }}$ May 2016. Accepted: $6^{\text {th }}$ July 2016.

Jeengar P, Chauhan M. Association of metabolic syndrome in polycystic ovarian syndrome. The New Indian Journal of OBGYN. 2017; 3(2): 90-4 
secretion of ovarian androgen but also promotes an increase in proportion of free (active) hormone.

It is well recognized that visceral distribution of body fat, common in syndrome is of greater consequences to the metabolic effect of insulin resistance than obesity per se. Central obesity and insulin resistance lead to an altered lipolytic response to insulin, with impaired suppression of release of free fatty acids from adipose tissues. An increased flux of free fatty acids from central sites enters the portal circulation, increasing the availability of substrate to the liver for VLDL- triglyceride production. Furthermore, women with the syndrome exhibit increased activity of hepatic lipoprotein lipase, an enzyme responsible for conversion of large lipoprotein particle to smaller more atherogenic particles. PCOS (Polycystic ovarian syndrome) is one condition commonly detected in a younger age group. PCOS affected patients may gain particular benefit from early screening for cardiovascular risk factor. So, this study was aim to observe the association of metabolic syndrome in Polycystic Ovarian Syndrome.

\section{Methods}

This is an observational study which is conducted at the Department of Obstetrics and Gynecology, RNT Medical College, Udaipur (Rajasthan) during the period of January 2015 to December 2015. Fifty apparently healthy non-pregnant females of 15-35 years having documented features of PCOS (according to Rotterdam criteria, 2003) are selected from those attending the outpatient department (OPD) and assessed for presence of metabolic syndrome (according to NCEP criteria) and compared with 50 women having normal pelvic study.

\section{Inclusion criteria-}

1) Cases - Women between 15-35 years with PCOS (according to Rotterdam criteria, 2003)

2) Control - Women between 15-35 years with normal pelvic study.

Exclusion criteria-

1) Diabetes

2) Hypertension

3) Any cardiovascular disease
Investigations: Age, weight, waist circumference and blood pressure were recorded.

Collection of blood samples: $5 \mathrm{ml}$ of venous blood was collected in heparinised bottle after an overnight fast of $12 \mathrm{hrs}$. Serum was separated and the following parameters were estimated - 1. Estimation of fasting blood sugar, 2. Estimation of total cholesterol by CHOD-PHOD/phosphor tungstate method of Alliance 16, 3. Estimation of High Density Lipoprotein (HDL) cholesterol by CHOD - POD phosphotungstate method by Burstein 17, 4. Estimation of triglycerides by glycerol phosphate oxidase method of Jacob 18.

Rotterdam PCOS diagnostic criteria [9]: Two out of three should be present $\quad$ r 1 ) Oligoovulation/anovulation (amenorrhea or oligomenorrhoea), 2) Clinical and/or biochemical signs of hyperandrogenism (hirsutism/ acne/ alopecia/ elevated levels of free or total testosterone) and 3) Polycystic ovaries on USG.

NCEP diagnostic criteria for metabolic syndrome [10]: Three out of five should be present - 1) Fasting plasma glucose $\geq 100 \mathrm{mg} / \mathrm{dl}$, 2) Systolic blood pressure $>130 \mathrm{~mm}$ of $\mathrm{Hg}$ or diastolic $\mathrm{BP}>85 \mathrm{~mm}$ of $\mathrm{Hg}, 3$ ) Waist circumference $>35$ inches, 4) Serum triglyceride $\geq 150 \mathrm{mg} / \mathrm{dl}$ and 5) HDL cholesterol $\leq 50 \mathrm{mg} / \mathrm{dl}$.

This study is approved by institutional ethics committee. Each patient had undergone a detailed clinical examination and a relevant laboratory evaluation. The data were collected in structured proforma and it was analyzed with relevant statistical methods.

\section{Results}

A group of 50 women having PCOD underwent detailed evaluation for presence of metabolic syndrome and compared with 50 women having normal pelvic study. Mean age of the patients who entered the study is $23.68 \pm 4.85$ years with maximum $(68 \%)$ patients in the age group of 15-25 years. Mean age of control group is $25.18 \pm 5.4$ years. Thus age of the patients in case group is slightly on lower side as compare to control group. Sixty six percent of PCOS patients were obese. Sixty percent of patients had typical appearance of PCO on ultrasonography. Clinical manifestation of 


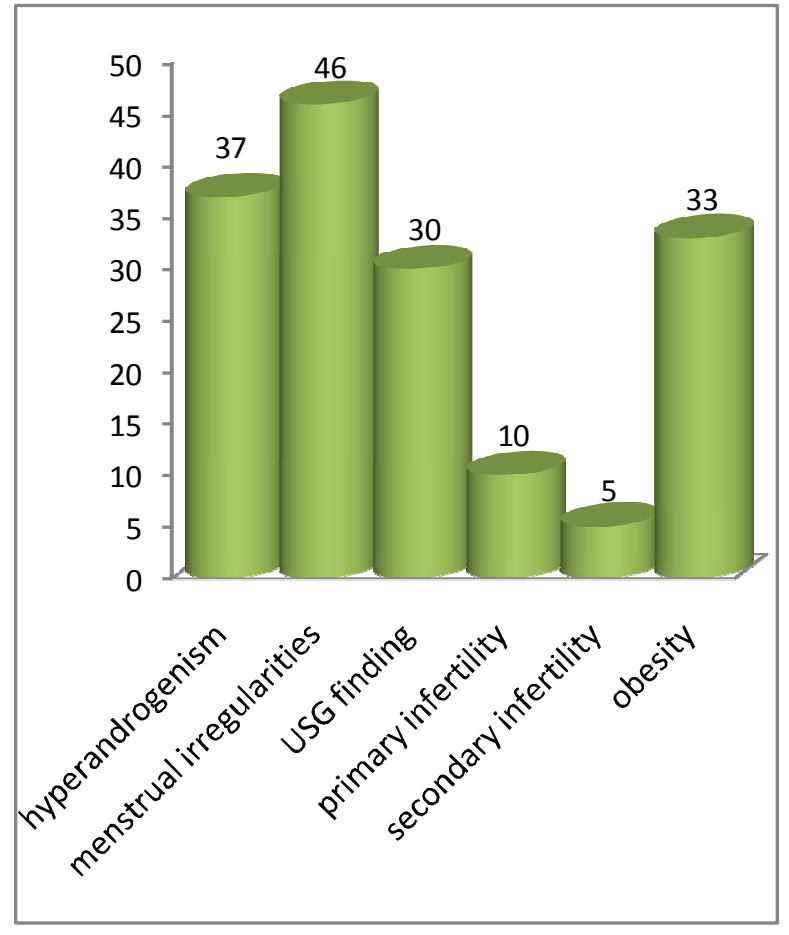

Figure 1: Clinical presentation of PCOS patients

hyperandrogenemia is seen in $34(74 \%)$ of PCOS patients. Oligo / amenorrhea is seen in 46 (92\%) of patients. Primary infertility is seen in $10(20 \%)$ and

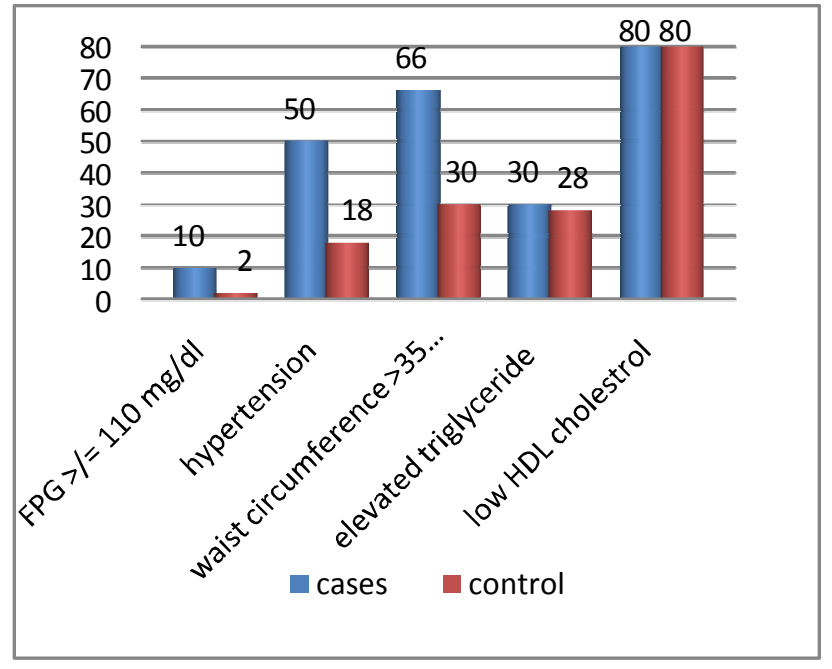

Figure 2: Comparison of components of metabolic syndrome secondary infertility in $5(10 \%)$ of cases (Figure 1).

Fifty eight percent of patients with PCOS show features of metabolic syndrome as compared to $36 \%$ patients of control group. Ten percent of PCOS patients show impaired glucose tolerance as compared to $2 \%$ of control group. Waist circumference $>35$ inches is seen in $66 \%$ of cases as compared to $30 \%$ patients in control group. Hypertriglyceridemia is seen in $30 \%$ and $28 \%$ patients of cases and control respectively. HDL - C $<50 \mathrm{mg} / \mathrm{dl}$ is seen in $80 \%$ patients of both groups (Figure 2).

Comparing the mean data for different variables, it was noted that waist circumference, systolic and diastolic blood pressures, fasting glucose had a significantly higher value among those with PCOS in comparison to those without the PCOS (table 1).

\section{Discussion}

In our study women with abdominal obesity i.e. increased waist circumference ( $>35$ inches) and ultrasound findings are taken as identifiable factors for detecting PCOS cases. Ultra-sound findings are similar in women with abdominal obesity and in a smaller group of lean patients with PCOS [11]. The results of our study indicate $58 \%$ prevalence of metabolic syndrome in PCOS. This is greater than the observations of $42 \%$ and $46 \%$ prevalence made by Ramprasad Dey et al [12] and Glueck CJ et al [13] respectively. Two other studies had also shown the prevalence of metabolic syndrome $47.3 \%$ (Ehrmann's et al, 2006) [14] and 33.4\% (Dokra's et al, 2005) [15] respectively among sampled women with PCOS. The age adjusted prevalence of metabolic syndrome has shown that women in between 15-25 yrs have higher prevalence $(68 \%)$ of metabolic syndrome, in comparison to only $32 \%$ in $26-35$ yrs age group. It may be because of a comparatively younger age group took part in our study as most of young girls presented to OPD with menstrual problems and married women with primary infertility with average duration of infertility of 2.4 years. Another very important factor for younger patients developing metabolic syndrome may be related to their life style. Dietary habits 
(consumption of fast food etc.), lack of exercise, sedentary life style etc make them prone to develop obesity in their early life which is the root cause of
In case of other components like HDL-C, triglycerides, blood pressure, fasting glucose concentrations individual prevalence corroborated with

\begin{tabular}{|l|l|l|l|}
\hline \multicolumn{4}{|c|}{ Table 1: Mean of different components of metabolic syndrome } \\
\hline Criteria & Cases & Control & P- Value \\
\hline Age (years) & $23.6 \pm 4.85$ & $25.18 \pm 5.4$ & - \\
\hline Waist circumferences (inches) & $35.98 \pm 3.49$ & $33.06 \pm 3.21$ & $\mathrm{P}<0.001$ \\
\hline SBP (mm of Hg) & $129.96 \pm 10.32$ & $123.92 \pm 7.5$ & $\mathrm{P}<0.001$ \\
\hline DBP (mm of Hg) & $83.32 \pm 8.06$ & $78.84 \pm 6.9$ & $\mathrm{P}<0.001$ \\
\hline Fasting blood sugar (mg/dl) & $95.96 \pm 11.95$ & $92.5 \pm 9.3$ & $\mathrm{P}<0.001$ \\
\hline Serum Triglyceride (mg/dl) & $145.21 \pm 26.89$ & $131.3 \pm 24.6$ & $\mathrm{P}<0.05$ \\
\hline HDL-C (mg/dl) & $42.67 \pm 7.90$ & $45.14 \pm 6.9$ & $\mathrm{P}<0.05$ \\
\hline P value $<0.05$ is considered statistically significant & \\
\hline
\end{tabular}

that observed by Glueck CJ et al and Ramprasad Dey et al. (table 2). Presence of a fasting plasma glucose of $110 \mathrm{mg} / \mathrm{dl}$ or greater was seen in $10 \%$ of cases which is in concordance with the study done by Glueck et al having $11 \%$ patients with high value of FBS. In control group this is seen in only $2 \%$ of patients. This suggests that impaired glucose tolerance secondary to insulin

development of both PCOS and metabolic syndrome. Life style playing a role in development of metabolic syndrome can also be explained by the fact that majority of patients who develop metabolic syndrome in our study belong to urban areas.

In our study waist circumference above the threshold of 35 inches was found in $66 \%$ cases which

\begin{tabular}{|l|l|l|l|}
\hline Table 2: Comparison with other studies \\
\hline Criteria & $\begin{array}{l}\text { Ramprasad } \\
\text { Dey et al }\end{array}$ & $\begin{array}{l}\text { Glucek } \\
\text { CJ et al }\end{array}$ & $\begin{array}{l}\text { Present } \\
\text { study }\end{array}$ \\
\hline Age (years) & $28.2 \pm 5.8$ & $31 \pm 9$ & $23.6 \pm 4.85$ \\
\hline Waist circumferences (inches) & $36.4 \pm 4.9$ & $45.6 \pm 5.6$ & $35.98 \pm 3.4$ \\
\hline SBP (mm of Hg) & 130 & $131 \pm 13$ & $129.9 \pm 10.3$ \\
\hline DBP (mm of Hg) & $87.8 \pm 3.3$ & $83 \pm 7$ & $83.32 \pm 8.06$ \\
\hline Fasting blood sugar (mg/dl) & $99.3 \pm 20.4$ & $94 \pm 22$ & $95.96 \pm 11.9$ \\
\hline Serum triglycerides (mg/dl) & $167.7 \pm 29.4$ & $192 \pm 152$ & $145.2 \pm 26.9$ \\
\hline HDL-C (mg/dl) & $42.6 \pm 6.8$ & $39 \pm 7$ & $42.67 \pm 7.90$ \\
\hline
\end{tabular}

resistance is one of the most important factors in development of PCOS. Thus PCOS become a high risk factor for development of metabolic syndrome.

$\mathrm{HDL}-\mathrm{C}$ values $<50 \mathrm{mg} / \mathrm{dl}$ was seen in $80 \%$ patients of both groups. This may be because most patients using saturated fatty acids in our locality. Hypertriglyceridemia is seen in $30 \%$ and $28 \%$ patients of cases and control respectively. This suggests that in our study dyslipidemia does not show much association with PCOS patients. This may be because of comparatively younger patients taking part in our study but this should also be taken into consideration that these patients are at high risk to develop metabolic syndrome in their later life. Factors other than insulin resistance may be implicated in pathogenesis of

was significantly higher than the control group (30\%). It is suggestive of obesity playing a major role in development of metabolic syndrome in PCOS patients. Twenty seven patients out of 33 obese patients show presence of metabolic syndrome. Development of obesity in younger age is again attributed to sedentary life style. Thus there is a strong need to aware the patients about life style modification at an early age. dyslipidemia and this may require further studies.

\section{Conclusion}

It is observed from the study that the prevalence of metabolic syndrome in women with PCOS is significantly greater than that in normal women. Thus there is an urgent necessity to assess the rising trend of metabolic syndrome among the women with PCOS and to take early measures for primary prevention of its long term sequel. 


\section{Conflict of interest: None. Disclaimer: Nil.}

\section{References}

1. Stein IF, Leventhal ML. Amenorrhoea associated with bilateral polycysticovaries. Am J Obstet Gynecol. 1935; 29: $181-91$

2. Legro RS, Kunselman AR, Dodson WC, Duanif A. Prevalence and predictors of risk for type 2 diabetes mellitus and impaired glucose tolerance in polycystic ovary syndrome: A prospective, controlled study in 254 affected women. J Clin Endocrinol Metab.1999; 84:16569.

3. Faloia E, Canibus P, Gatti C, Frezza F, Santangelo M, Garrapa GG, et al. Body composition, fat distribution and metabolic characteristics in lean and obese women with polycystic ovary syndrome. J Endocrinol Invest. 2004; 27: 424-9.

4. Crossgnanil PG, Nicolosi AE. Polycystic ovarian disease: Heritability and heterogenicity. Hum report. 2001; 7: 3-7

5. Franks S, Gilling-Smith C, Gharani N, McCarthy M. Pathogenesis of polycystic ovary syndrome: evidence for a genetically determined disorder of ovarian androgen production. Hum Fertil. 2000; 3: 77-9.

6. Gharani N, Waterworth DM, Batty S, et al. Association of the steroid synthesis gene CYP11a with polycystic ovary syndrome and hyperandrogenism. Hum Mol Gen. 1997; 6: 397-402

7. Waterworth DM, Bennett ST, Gharani N, et al. Linkage and association of insulin gene VNTR regulatory polymorphism with polycystic ovary syndrome. Lancet. 1997; 349: 986-90.

8. Steppan CM, Bailey ST, Bhat S, et al. The hormone resist in links obesity to diabetes. Nature. 2001; 409: 30712
9. The Rotterdam ESHRE/ASRM-Sponsored PCOS Consensus Workshop Group. Revised 2003 consensus on diagnostic criteria and long-term health risks related to polycystic ovary syndrome. Fertil Steril. 2004; 81:19-25

10. Executive Summary of the Third Report of the National Cholesterol Education Program (NECP), Expert Panel on Detection, Evaluation and Treatment of High Blood Cholesterol in Adults (Adult Treatment Panel III). Journal of the American Medical Association. 2001; 285: 2486-97.

11. Canibus P, Gatti C, Frezza F, Santangelo M, Garrapa $\mathrm{GG}$, et al. Body composition, fat distribution and metabolic characteristics in lean and obese women with polycystic ovary syndrome. J Endocrinol Invest. 2004; 27 : 424-9

12. Dey R, Mukherjee S, Roybiswas R, Mukhopadhyay A, Biswas SC. Association of metabolic syndrome in polycystic ovarian syndrome. J Obstet Gynecol India. 2005; 61:176-81.

13. Glueck CJ, Papanna R, Wang P, Goldenberg N, Sieve-Smith L. Incidence and treatment of metabolic syndrome in newly referred women with confirmed polycystic ovary syndrome. Metabolism. 2003; 52: 908 15.

14. Ehrmann DA, Liljenquist DR, Kasza K, et al. Prevalence and predictors of the metabolic syndrome in women with polycystic ovary syndrome. J Clin Endocrinol Metab. 2006; 91: 48-53.

15. Dokras A, Bochner M, Hollinrake E, et al. Screening women with polycystic ovary syndrome for metabolic syndrome. Obstet Gynecol. 2005; 106: 131-37.

\footnotetext{
Pooja Jeengar ${ }^{1}$, Madhubala Chauhan ${ }^{2}$

${ }^{1}$ PG student in Department of Obstetrics and Gynecology, R.N.T. Medical College, Udaipur, Rajasthan, India; ${ }^{2}$ Senior Professor in Department of Obstetrics and Gynecology, R.N.T. Medical College, Udaipur, Rajasthan, India
} 